\title{
Immunohistochemical study of subepidermal connective of molluscan integument
}

\author{
S. Corbetta, A. Bairati, and L. Vitellaro Zuccarello
}

Dipartimento di Fisiologia e Biochimica Generali, sezione di Istologia ed Anatomia Umana, Università degli Studi di Milano, via Celoria 26, 20133 Milano, Italy.

Accepted: 02/04/02

Key words: collagen, laminin, nidogen, proteoglycans, integument, Mollusca, immunohistochemistry

\section{SUMMARY}

Sections of integument from gastropod, bivalve and cephalopod species were studied immunohistochemically to determine reactivity to antibody against the type I-like collagen from Sepia cartilage and antibodies against components of the extracellular matrix (ECM) of vertebrate connective tissue: type I, III, IV, V, and VI collagens, laminin, nidogen and heparan sulphate. All samples exhibited similar reactivities to the antibodies, although differences in the intensity and localization of the immunostaining were found that were clearly correlated with between-species differences in integumental ultrastructure. These findings indicate that the composition of the integumental ECM is similar in the three classes of molluscs examined and that several types of collagen are present. However molluscan subepidermal connective tissue differs from the ECM of vertebrate dermis: molluscan integumental ECM contains collagens similar to type I, V and VI collagens but has no type III-similar collagen. Furthermore molecules similar to the type IV collagen, laminin, nidogen and heparan sulphate of vertebrates were present ubiquitously in molluscan basement membrane, confirming the statement that the structure and composition of basement membrane have remained constant throughout the evolution of all animal phyla.

\section{INTRODUCTION}

Interdisciplinary research has demonstrated an extraordinary variety of molecules in vertebrate integument, and is elucidating the roles that the interactions of these molecules play in fulfilling integumental functions. The dermis of mammals, for example, is a highly differentiated tissue whose extracellular matrix (ECM) is composed of numerous types of proteoglycans, glycoproteins and particularly collagens, which aggregate in various and complex ways to give rise to characteristic ECM structures: the basement membrane (type IV collagen), heterotypic fibrils (type I, III, V, XII and XIV collagens), microfilaments (type VI collagen) and anchoring fibrils (type VII collagen) (Keene et al., 1997).

In view of the great variety of invertebrate integuments, and our limited knowledge of their molecular components, we decided to carry out a comparative study of the integuments of several species of molluscs from classes of diverse evolutionary development, to determine whether or not they were made up of molecules similar to those present in 
mammalian dermis. Since elastin, an essential component of vertebrate ECM, seems to be absent from molluscan connective tissue (Sage and Gray, 1979), we concentrated our attention on collagens, certain other glycoproteins, and proteoglycans.

The molluscs are an ancient and successful phylum. Fossils reliably identified as molluscan are found in Precambrian strata, and today it is the second largest animal phylum, with marine, freshwater and terrestrial forms, with a gradation of evolutionary and structural diversity that ranges from the Monoplacophora and Cephalopoda. It is likely, therefore, that the connective tissues from different molluscan forms will vary markedly.

Knowledge of the collagens present in molluscan integument is limited, as is our knowledge of molluscan connective tissue components in general (Bairati, 1985). Kimura et al. (1981) isolated the main collagen present in the integument of Octopus vulgaris and characterized it as an $\left(\alpha_{1}\right)_{2} \alpha_{2}$ heterochain molecule similar to the type I collagen of vertebrates. Ultrastructural studies on the integument of various species of mollusc (Bairati et al., 2000; Bairati et al., 2001; Brocco, 1976) have demonstrated the presence of a basement membrane, banded collagen fibrils and proteoglycan aggregates. To determine whether these morphological components, typical of vertebrate dermis, also have molecular structures analogous to those of vertebrates, we performed the present immunohistological study on sections of integument from gastropod, bivalve and cephalopod species, using antibodies against components of vertebrate dermis. These studies were based on ultrastructural observations, in part published elsewhere (Bairati et al., 2000; Bairati et al., 2001), necessary for the control and interpretation of the immunohistochemical results.

\section{MATERIALS AND METHODS}

\section{Tissue preparation}

Samples of integument were obtained from the mantle and foot of four gastropods, the marine limpet Patella ulyssiponensis, the freshwater snail Pila scutata, the terrestrial slug Arion rufus, the terrestrial snail Helix aspersa, and three marine bivalves, Pecten jacobaeus, Mytilus galloprovincialis and Callista chione. Mantle, tentacle and arm samples were also obtained from the cephalopod cuttlefish Sepia officinalis. In all cases samples were removed from the animals immediately after capture.

Specimens for cryo-sectioning were fixed in either $10 \%$ formalin or $4 \%$ paraformaldehyde (PFA) in $0.1 \mathrm{M}$ phosphate buffer (PB) pH 7.4 or periodate-lysine-paraformaldehyde (PLP) in $0.1 \mathrm{M}$ $\mathrm{PB}$ pH 7.4 for various times (3-12h) depending on size. Subsequently the samples were impregnated in sucrose and fragments were then embedded in OCT compound, frozen in liquid nitrogen or isopentane cooled with liquid nitrogen and cryostat sectioned $(10 \mu \mathrm{m})$. Cryostat sections were also prepared from fragments of frozen unfixed tissue. To check morphology, sections adjacent to immunostained sections were stained with $1 \%$ methylene blue.

Specimens for conventional sectioning were fixed in 4\% PFA or Bouin's fluid for 24-48h, dehydrated, embedded in paraffin and sectioned (7 $\mu \mathrm{m})$. To check morphology, sections adjacent to immunostained sections were stained with hematoxylin and eosin.

\section{Enzyme pretreatment}

When antigen unmasking was required, principally to obtain a reaction on basement membrane of Sepia samples, paraffin embedded or frozen sections were treated with $1 \mathrm{mg} / \mathrm{ml}$ pepsin $(2100$ units/mg dry solid; Sigma) in $0.01 \mathrm{~N} \mathrm{HCl}$ at $37^{\circ} \mathrm{C}$ for 10-60 min (Wakamatsu et al., 1997).

\section{Antibodies}

We used the following antibodies, whose specificity for the respective antigens has been accurately tested as described either in the papers subsequently quoted for each antibody or in the data sheets supplied by the producers: polyclonal antibodies against type I-like collagen from Sepia officinalis cartilage (Bairati et al., 1999), goldfish type I collagen (Zylberberg et al., 1992), human type I (Démarchez et al., 1987), III and IV (Institut Pasteur, Lyon) collagens, rat type V collagen (ViallePresles et al., 1989); polyclonal antibodies against mouse EHS (Sigma) and rat (Gibco) laminin; monoclonal antibodies against human type VI collagen (clone C-1, Seikagaku), murine nidogen (Chemicon), the sugar chain of human heparan sulfate (clones HepSS-1 and F58-10E4, Seikagaku), and 
the protein portion of rat heparan sulfate $(\mathrm{C} 17$, Hybridoma Bank). The optimal working dilution was determined empirically for each antibody.

\section{Indirect immunofluorescence}

Sections of fixed samples were treated with $0.05 \mathrm{M}$ $\mathrm{NH}_{4} \mathrm{Cl}$ in $0.01 \mathrm{M}$ PBS to neutralize aldehyde and then incubated with 1-10\% PBS/bovine serum albumin (BSA) for $1 \mathrm{~h}$ at room temperature. Subsequently primary antibodies, diluted in $0.1 \%$ PBS/BSA, were applied overnight at $4^{\circ} \mathrm{C}$. The secondary antibodies, either FITC (Fluorescein Isothiocyanate) or Alexa Fluor 488 or Rhodamine Red-X or LRSC (Lissamine Rhodamine B Sulfonyl Chloride) conjugated, diluted in $0.1 \% \mathrm{PBS} / \mathrm{BSA}$, were applied for $1 \mathrm{~h}$ at room temperature. On control sections BSA was used instead of either primary antibody or both primary and secondary antibodies.

Sections were examined under a Leica DMR microscope equipped for epifluorescence with excitation filters BP450-490 and BP 546/14 and barrier filters LP 520, BP 525/520 and LP 580 Photomicrographs were taken with a MC 100 SPOT microscope camera.

\section{Immunoperoxidase}

Endogenous peroxidase activity was blocked by incubation with $0.3 \% \mathrm{H}_{2} \mathrm{O}_{2}$ in methanol for $30 \mathrm{~min}$. Aldehyde groups were neutralized by $1 \mathrm{~h}$ incubation in $0.05 \mathrm{M} \mathrm{PBS} / \mathrm{NH}_{4} \mathrm{Cl}$. The sections were then incubated with $\mathrm{PBS} / \mathrm{BSA}$ for $1 \mathrm{~h}$ at room temperature, followed by overnight incubation at $4^{\circ} \mathrm{C}$ with primary antibody, diluted in $0.1 \%$ PBS/BSA. Biotinylated secondary antibodies, in $0.1 \%$ PBS/BSA, were then applied for $2 \mathrm{~h}$ at room temperature, followed by $30-60 \mathrm{~min}$ incubation in avidin/biotinylated horseradish peroxidase complex (Vector) at room temperature. The immunoreaction was visualized with a solution containing $0.075 \%$ 3,3'-diaminobenzidine (Sigma) in $0.05 \mathrm{M}$ TRIS/ $\mathrm{HCl}$ and $0.2 \%$ $\mathrm{H}_{2} \mathrm{O}_{2}(10 \mu \mathrm{l} / \mathrm{ml})$. On control sections BSA was used instead of either primary antibody or both primary and secondary antibodies. Sections were examined under a Zeiss photomicroscope.

\section{RESULTS}

The immunostaining results are summarized in Table I. None of the control sections showed clear positivity for any antibody, however a certain amount of weak autofluorescence was observed in several control sections from various species which we attributed to the presence of pigments and secretions: these were often observed in gland cells, epidermal mucocytes, pigment cells and along the epidermal surface (Fig. 2D, 5B, 5F). In general the antibodies revealed, with variable staining intensity, structures of variable form and localization within the integument. Very often there was a meshwork of positivity within the matrix of the subepidermal connective (SEC), occasionally thickened into clumps or irregularly branching forms. Often too the positivity defined compact laminae, mainly present within the SEC at the base of the epithelium or surrounding ducts, chromatic elements, blood vessels, nerves and muscles fibres.

We now present the results by antibody for each species.

\section{Anti-Sepia type I-like collagen antibody}

Antibody against type I-like collagen from Sepia cartilage gave an intense meshwork of positivity throughout the SEC of all the gastropods examined; this was particularly intense under the epithelium, surrounding glands (particularly numerous in gastropod integument) and surrounding bundles of muscle fibres (Fig. 1A). In foot and mantle of all the bivalves investigated both the superficial and deep SEC showed an intense meshwork of positivity (Fig. 3A). In Pecten and Mytilus the immunostaining was particularly conspicuous in the areas surrounding muscles and glands, while in the foot of Callista the gland-rich zone separating the SEC from underlying muscle was picked out. As expected, the matrix of the SEC of Sepia reacted strongly to this antibody, as did stroma between bundles of muscle fibres (Fig 5A).

\section{Anti-fish type I collagen antibody}

The distribution of positivity to antibody against fish type I collagen in the integument of the gastropods Patella and Pila was closely similar to that described for antibody against Sepia type I-like collagen. In Helix however the positivity was weaker and at the same time more diffuse, and in Arion (Fig. 1B) positivity was diffuse and weak immediately under the epithelium, but stronger at depth.

The integument of the bivalves studied also reacted to anti-fish type I collagen antibody. In Mytilus (Fig. 3B) the reaction was less intense 
Table I

Results of the immunohistochemical analyses on molluscan integument

CLASS and SPECIES

\begin{tabular}{|c|c|c|c|c|c|c|c|c|}
\hline \multirow[b]{2}{*}{ Antibodies } & \multirow[b]{2}{*}{$\begin{array}{c}P . \\
\text { scutata }\end{array}$} & \multicolumn{2}{|c|}{ GASTROPODA } & \multirow[b]{2}{*}{ H. aspersa } & \multirow[b]{2}{*}{$\begin{array}{l}\text { P. jaco- } \\
\text { baeus }\end{array}$} & \multirow{2}{*}{$\begin{array}{l}\text { BIVALVIA } \\
\text { M. gallo- } \\
\text { provincialis }\end{array}$} & \multicolumn{2}{|c|}{ CEPHALOPODA } \\
\hline & & $\begin{array}{l}\text { P. ulyssi- } \\
\text { ponensis }\end{array}$ & A. rufus & & & & C. chione & S. officinalis \\
\hline anti-cuttlefish type & & & & & & & & \\
\hline I-like collagen & + & + & + & + & + & + & + & + \\
\hline $\begin{array}{l}\text { anti-fish type I collagen } \\
\text { anti-human type I }\end{array}$ & + & + & + & + & + & + & + & + \\
\hline $\begin{array}{l}\text { collagen } \\
\text { anti-human type III }\end{array}$ & n.e. & n.e. & - & - & - & - & - & - \\
\hline collagen & n.e. & n.e. & - & - & - & - & - & - \\
\hline $\begin{array}{l}\text { anti-rat type V collagen } \\
\text { anti-human type VI }\end{array}$ & + & + & + & + & + & + & + & + \\
\hline $\begin{array}{l}\text { collagen } \\
\text { anti-human type IV }\end{array}$ & + & + & + & + & + & + & + & + \\
\hline collagen & + & + & + & + & + & + & + & + \\
\hline anti-rat/mouse laminin 1 & + & + & + & + & + & + & + & + \\
\hline $\begin{array}{l}\text { anti-murine nidogen } \\
\text { anti-rat HSPG proteinous }\end{array}$ & + & + & + & - & + & + & + & + \\
\hline $\begin{array}{l}\text { portion } \\
\text { anti-human HSPG }\end{array}$ & n.e. & n.e. & - & + & - & + & - & - \\
\hline sugar chain & + & + & + & + & + & + & + & + \\
\hline
\end{tabular}

n.e.= not examined

than that obtained with antibody against Sepia type I-like collagen; in Pecten and Callista positivity was distributed fairly uniformly throughout the SEC, although the glandular layer was less reactive to this antibody than to those against other types of collagen.

In Sepia this antibody produced a reaction similar to that observed with anti-Sepia type I-like collagen antibody except that the epidermis itself was also stained (Fig. 5C).

\section{Antibodies to human type I and III collagens}

These antibodies did not elicit positivity in any of the integument samples studied.

\section{Anti-rat type $V$ collagen antibody}

In Pila and Patella treatment with antibody against rat type $\mathrm{V}$ collagen produced a labelling similar to that obtained with antibody against Sepia type I-like collagen. In Arion (Fig. 1C) and Helix the signal was weaker and more diffuse and similar to that observed with antibody against fish type I collagen. In the bivalve Pecten the entire SEC was intensely positive. In Mytilus the staining particularly involved the most superficial part of the SEC, being less intense in the deeper part (Fig. 3C). In Callista intense positivity was observed in connective tissue immediately enveloping glands.

In Sepia staining was intense superficially, much less so in the areas surrounding muscle fibres (Fig. 5D).

\section{Anti-human type VI collagen antibody}

The integument of the gastropods examined reacted very weakly with antibody against human type VI collagen. In Arion (Fig. 1D), Helix and Pila there was a weak meshwork of positivity in the superficial SEC that became more marked around blood vessels, muscle fibres, ducts and glands whose secretory contents were also often 

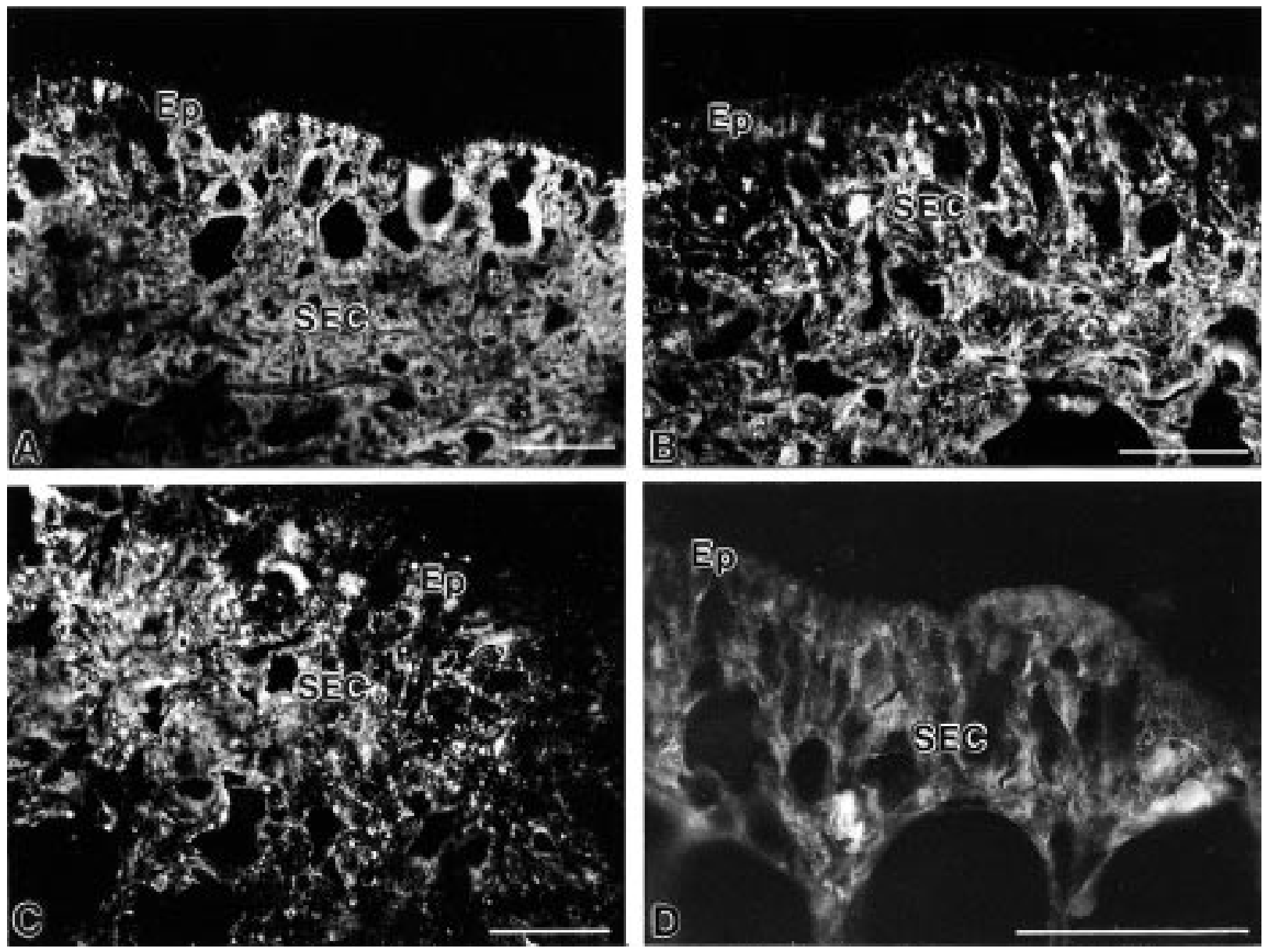

Fig. 1 - Indirect immunofluorescence staining of frozen sections of integument from the foot (A-C) and mantle (D) of the gastropod Arion rufus. (A) polyclonal anti-type I-like collagen from cuttlefish cartilage. (B) polyclonal anti-fish type I collagen. (C) polyclonal anti-rat type V collagen. (D) monoclonal anti-human type VI collagen. Ep, epidermis; SEC, subepidermal connective tissue. Bar, $100 \mu \mathrm{m}$.

strongly positive. In Patella the positivity was particularly evident around all structures embedded within the connective tissue.

The integument of the bivalve Pecten (Fig. 3D) was characterized by an intense and uniform ribbon of positivity under the epithelium. This diminished with depth and took on a network-like appearance. The staining in Mytilus was similar except that overall intensity was less. In Callista positivity was mainly concentrated around structures within the connective tissue, and was present as a weak network between muscle fibres.

In Sepia integument, positivity to antibody against human type VI collagen was similar to that for rat collagen $\mathrm{V}$ antibody (Fig. 5E) with intense positivity close to the epithelium that was consid- erably attenuated in the areas surrounding muscle fibres and also SEC areas surrounding other structures such as (autofluorescent) glands and chromatic elements (Fig. 5F).

\section{Anti-human type IV collagen antibody}

The antibodies against components of vertebrate basement membrane reacted positively with integument sections of all molluscs examined.

In Patella (Fig. 2A) treatment with anti-human type IV collagen antibody produced a weak reaction in the zone between the epithelium and the underlying connective tissue. This zone did not have a laminar appearance but expanded irregularly into the SEC. A similarly weak reaction was observed around ducts, glands, blood vessels, nerves, and muscle fibres, but was formed of short 

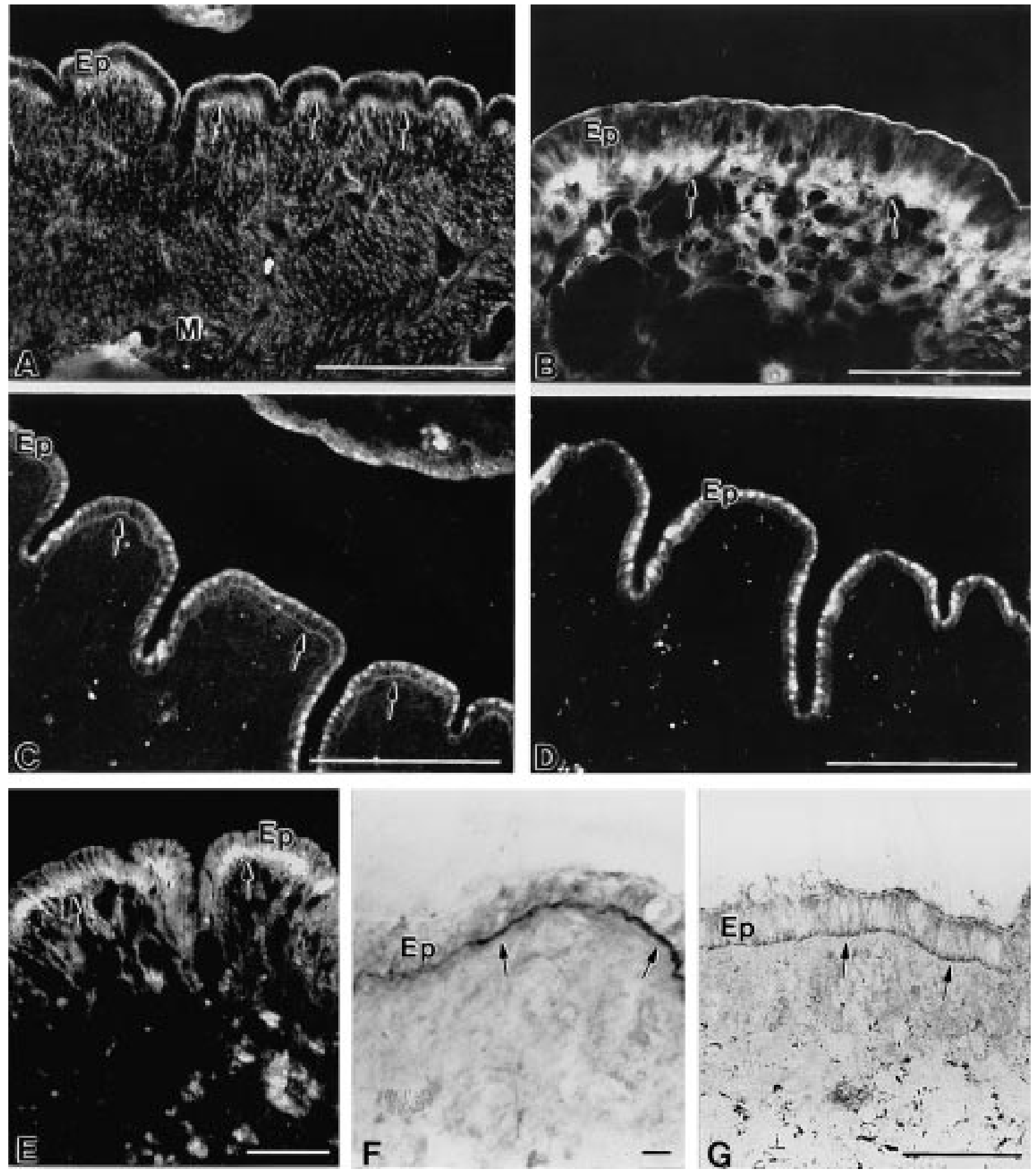

Fig. 2 - Indirect immunofluorescence and immunoperoxidase staining of frozen sections of gastropod foot integument with antibodies against BM components. Patella ulyssiponensis (A) and Arion rufus (B) integument stained with polyclonal anti-human type IV collagen. (C, D) Patella ulyssiponensis integument stained with monoclonal anti-murine nidogen and control section omitting primary antibody. (E) Helix aspersa integument stained with monoclonal antibody against the protein portion of rat HSPG. (F) Pila scutata integument stained with antibody against the sugar chain of HSPG. (G) Patella ulyssiponensis integument stained with polyclonal anti-mouse laminin. Ep, epidermis; M, muscle; arrows, basement membrane. Bar, $10 \mu \mathrm{m}$ in F; 100 $\mu \mathrm{m}$ in A-E and G. 

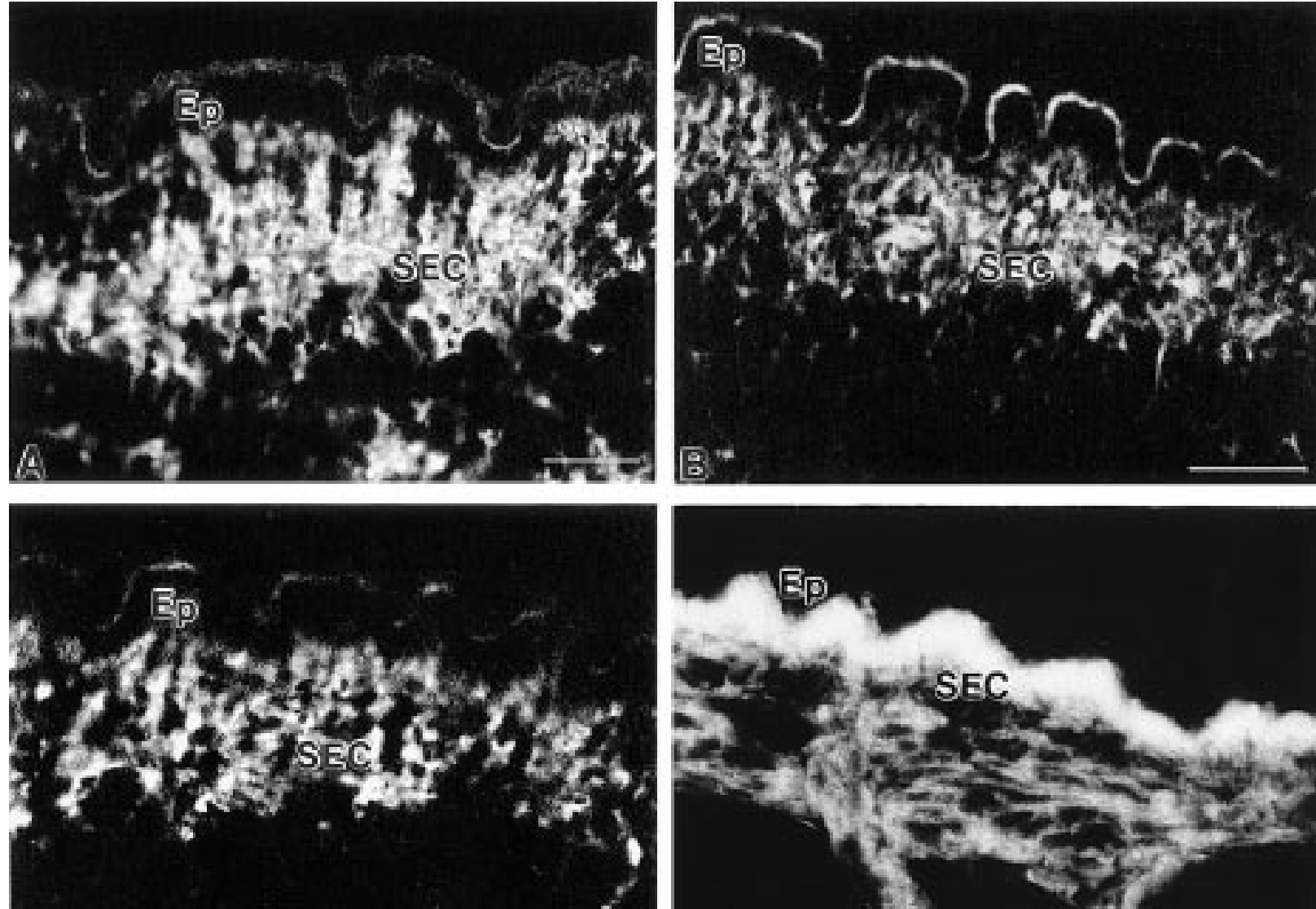

Fig. 3 - Indirect immunofluorescence staining of frozen sections of bivalve integument with anti-collagen antibodies. Mytilus galloprovincialis foot integument stained with polyclonal antibodies against cuttlefish type I-like (A), fish type I (B) and rat type V (C) collagens. (D) Pecten jacobaeus mantle integument stained with monoclonal anti-human type VI collagen. Ep, epidermis; SEC, subepidermal connective; M, muscle. Bar, $100 \mu \mathrm{m}$.

discontinuous tracts. In Arion (Fig. 2B) and Helix a sub-epithelial laminar reaction was not observed, while the SEC positivity was diffuse but occasionally concentrated into irregular clumps. In Pila there was discontinuous positivity in contact with the epithelium and a diffuse but irregular positivity in the rest of the SEC.

In Pecten (Fig. 4A) and Mytilus treatment with antibody against human type IV collagen revealed an intensely positive laminar structure immediately under the epithelium. In Callista a similar intensely positive laminar structure was also observed, from which ran short irregular protrusions into the SEC.

In Sepia a similar intense positivity picking out basement membrane was observed only in sections pre-treated with pepsin. As shown in Fig. 6A (immunoperoxidase) there was a thin but continuous line of positivity immediately below the epithelium, with occasional short protrusions into the connective tissue; the epidermis was also uniformly stained.

\section{Anti-laminin antibody}

The immunoperoxidase method localized laminin more clearly than indirect immunofluorescence on integumental sections from Patella (Fig. 2G) and Pila where a thin, discontinuous strip of laminin positivity was observed immediately below the epithelium and surrounding the various structures present within the SEC. In sections from Arion and Helix a clear sub-epithelial signal was not observed, but a weaker diffuse positivity was present surrounding glands and muscle fibres.

Laminin positivity was observed immediately beneath the epithelium of all bivalve sections examined. This positivity was intense in Pecten 

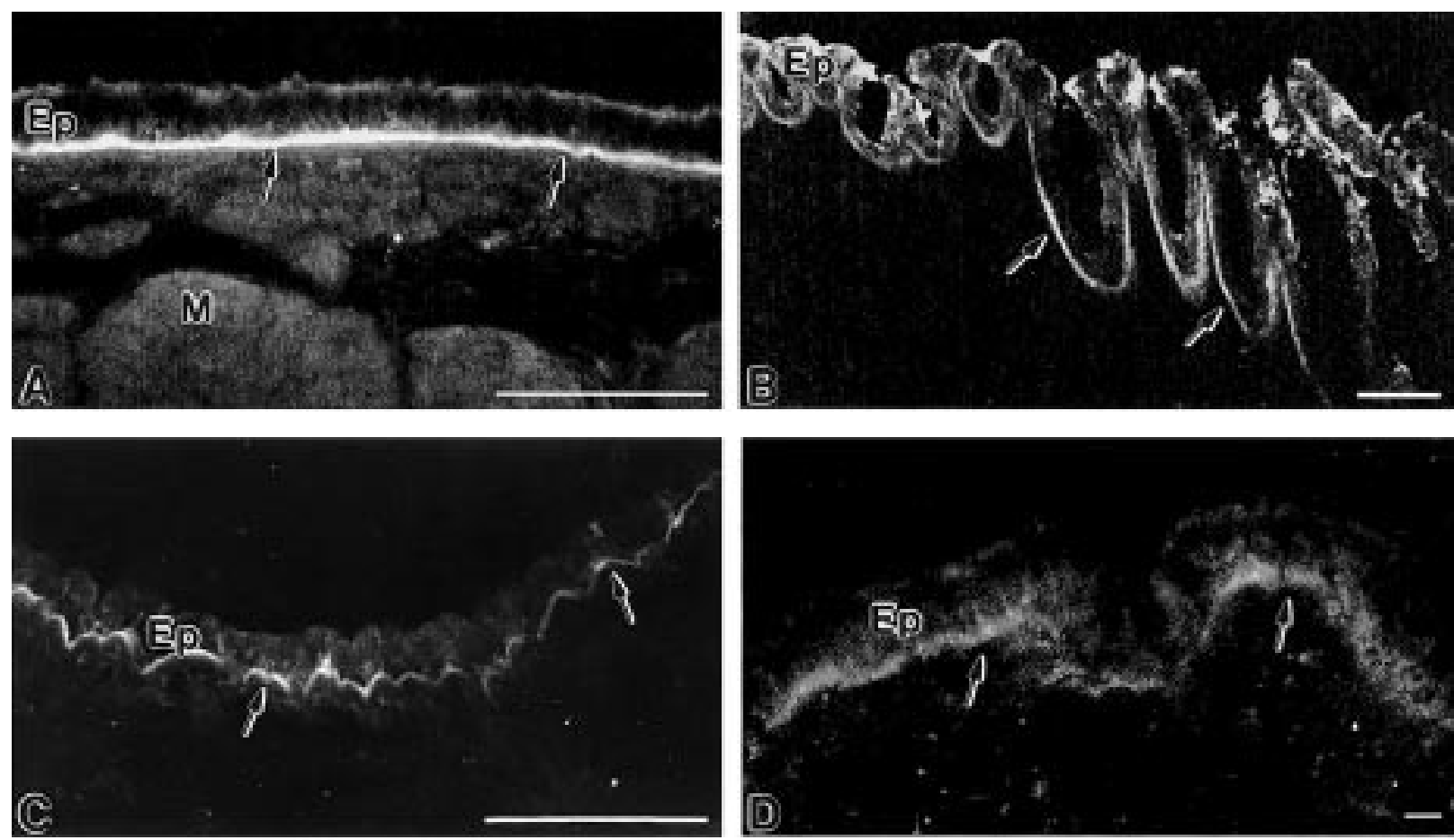

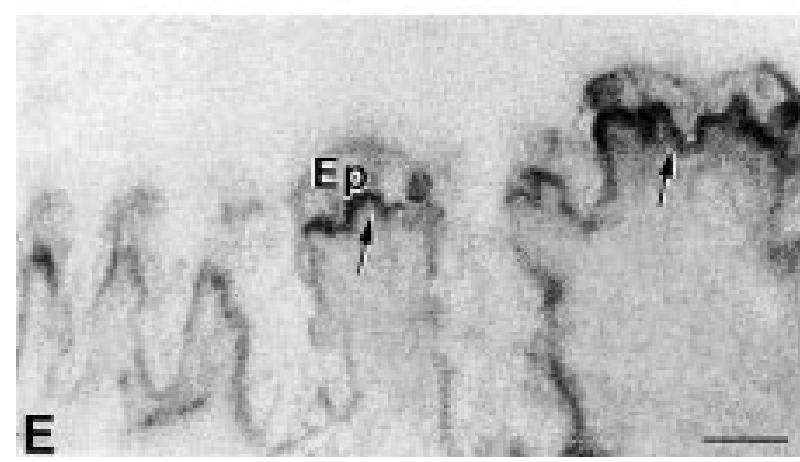

(Fig. 4B) but in Callista took the form of a thin discontinuous line. In Mytilus and Callista there was also positivity surrounding the structures with SEC. The Sepia sections, pre-treated with pepsin, present a thin line of laminin positivity below the epithelium and as intense surrounding structures within the SEC (Fig. 6B).

\section{Anti-mouse nidogen antibody}

Use of antibody against nidogen produced weak diffuse positivity throughout the SEC but no particular localization under the epithelium in Helix. In Arion there was increased sub-epidermal intensity; in Patella (Fig. 2C) a thin line of sub-epithelial positivity, and in Pila a discontinuous subepithelial signal.
Fig. 4 - Indirect immunofluorescence and immunoperoxidase staining of frozen sections of bivalve integument with antibodies against basement membrane components. Pecten jacobaeus mantle integument stained with (A) polyclonal anti-human type IV collagen, (B) polyclonal anti-rat laminin and (C) monoclonal anti-murine nidogen. (D) Mytilus galloprovincialis foot integument stained with monoclonal antibody raised against the protein portion of rat HSPG. (E) Callista chione mantle integument stained with antibody against the sugar chain of HSPG. Ep, epidermis; M, muscle; arrows, basement membrane. Bar, $10 \mu \mathrm{m}$ in D and E; 100 um in A-C.

In all bivalves sections examined (Fig. 4C), and also in Sepia sections (Fig. 6D), anti-nidogen antibody reaction always localized in the immediate sub-epithelial zone and around other structures within the SEC.

\section{Anti-heparan sulfate proteoglycan (HSPG) antibodies}

Antibody against the HSPG sugar chains localized to the sub-epidermal zone in all species examined. Staining was intense in Pila particularly when the immunoperoxidase method was used (Fig. 2F); in Patella a thin laminar zone was picked out, and in Arion and Helix the subepidermal immunostaining was discontinuous and irregular. The antibody against the protein portion of HSPG was tested on 

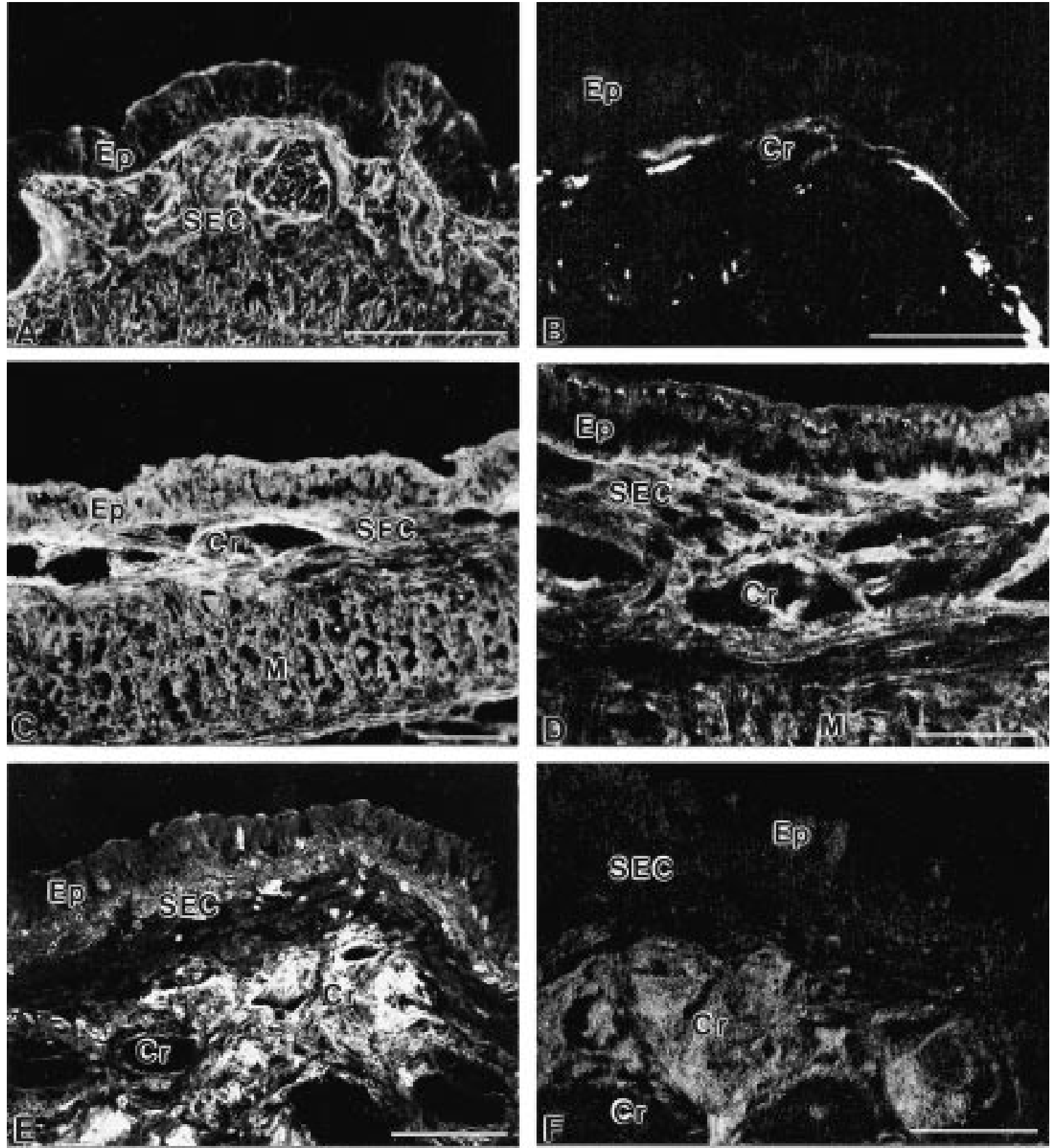

Fig. 5 - Indirect immunofluorescence staining of frozen (A-D) and paraffin (E, F) sections of the integument of Sepia officinalis (cephalopod) with anti-collagen antibodies. (A, B) Arm integument stained with polyclonal anti-cuttlefish type I collagen and control section omitting primary antibody. Mantle integument stained with polyclonal anti-fish type I (C) and anti-rat type V (D) collagens. Tentacle integument stained with monoclonal anti-human type VI collagen (E) and control section (F) omitting primary antibody: the autofluorescence of chromatic elements was easily seen. Ep, epidermis; SEC, subepidermal connective; M, muscle; $\mathrm{Cr}$, chromatic elements. Bar, $100 \mu \mathrm{m}$.

the gastropods Arion and Helix but only in the latter was positivity found. In Helix the reactivity localized strongly immediately under the epithelium, in protrusions from the sub-epithelial zone into the SEC, and within gland cells (Fig. 2E).
The sub-epithelial zone of all bivalves studied reacted positively to antibody against the HSPG sugar (Fig. 4E); only in Mytilus did this zone also react with the antibody against protein portion of HSPG (Fig. 4D). 

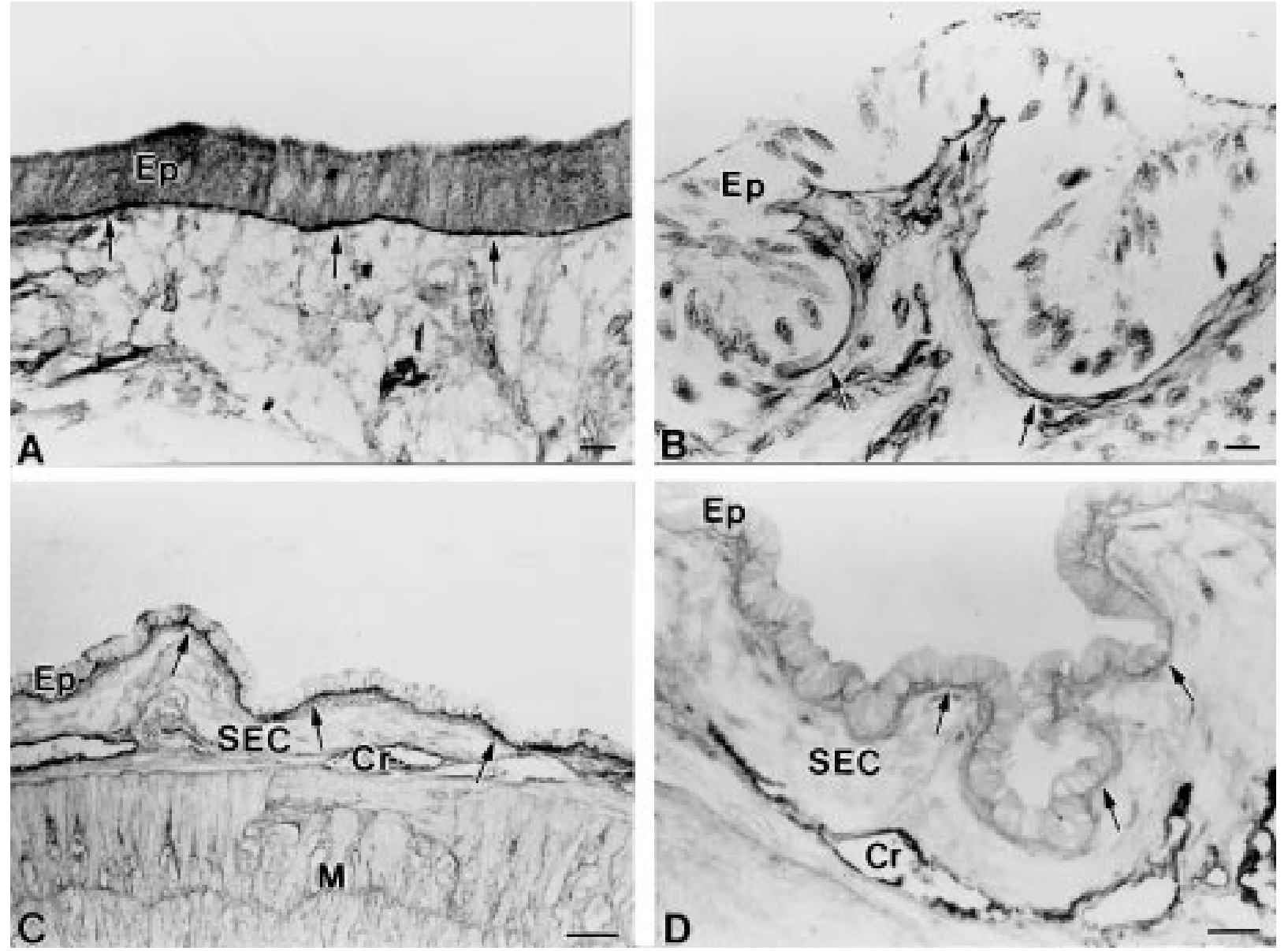

Fig. 6 - Immunoperoxidase staining of paraffin (A, B) and frozen sections (C, D) of Sepia officinalis integument with antibodies against basement membrane components. Arm integument stained with polyclonal anti-human type IV collagen (A) and antimouse laminin (B) after pepsin digestion. Mantle integument stained with monoclonal antibody against the sugar chain of HSPG (C) and anti-murine nidogen (D). Ep, epidermis; SEC, subepidermal connective; M, muscle; arrows, basement membrane; $\mathrm{Cr}$, chromatic elements. Bar, $10 \mu \mathrm{m}$ in A and B; $100 \mu \mathrm{m}$ in C and D.

In Sepia (Fig. 6C) all structures within SEC were clearly delineated by a rim of positivity with antibody against the carbohydrate moiety of human HSPG. The basement membrane zone and the zone surrounding muscle fibres was also labeled by this antibody. No clear signal was observed in Sepia when the antibody against the protein portion of rat HSPG was used.

\section{DISCUSSION}

This study has demonstrated that the integumental connective tissues of all the mollusc species examined present specific immunoreactivity to a range of antibodies against several of the mole- cules typically present in mammalian dermis. However both the intensity and localization of the reactivity to the different antibodies varied according to the mollusc class and species examined, and this variability is related to the diversity of ultrastructural organization present in these animals as revealed by our previous study (Bairati et al., 2000; Bairati et al., 2001). The latter studies showed that the thickness and detailed structure of the basement membrane, and the extent and looseness of the underlying connective tissue varied considerably among the species examined. These structures were most similar in species of the same class, and in the bivalves and gastropods, but differed more markedly between cephalopods and the other two classes. 
The meshwork of immunopositivity within the SEC was due to the presence of connective tissue containing a network of collagen fibrils. This network was rather loose or open in gastropods (Bairati et al., 2001) and bivalves (Bairati et al., 2000) but much denser in cephalopods where in fact bundles of collagen fibrils formed large fibres (Brocco, 1976; Bairati et al., in preparation). The SEC of the bivalve Pecten differed in that it consisted of a homogenous layer of tightly packed small collagen fibrils (Bairati et al., 2000) and the corresponding immunoreactivity was more diffuse and uniform. The compact laminar positivity present around glands, blood vessels, nerves, and chromatic elements, correlates with the presence of basement membrane and of a laminar arrangement of collagen fibrils surrounding these structures.

In all species the SEC reacted to antibodies against type I, type V and type VI collagens, confirming the ubiquitous presence of these molecules (or molecules with closely similar epitopes) within molluscan integumental tissue. Kimura et al. (1981) showed that the most abundant collagen in the integument of Octopus vulgaris is a heterochain molecule that has certain characteristics in common with calf type I collagen and was therefore called type I-like. The intense SEC reaction, in all the species studied, to the antibody against type I-like collagen from Sepia cartilage shows that the collagen in the integumental SEC of all these species is closely similar to that in Sepia cartilage, suggesting that such a collagen is present in connective tissues of various organs throughout the phylum.

The present study also revealed the presence, in the SEC of molluscs, of a collagen having immunological affinity to type I collagen from fish. However there was never any positivity to anti-human type I collagen antibody. This affinity is consistent with the fact that both fish and mollusc collagens have lower denaturation temperatures than mammalian collagens, in relation lower environmental temperature (see discussion in Mathews, 1975a). This is related to the presence of fewer pyrrolidinic cross-links in collagens from fish and molluscs, implying a molecular structure distinct from that of type I mammalian collagen. We may discern an evolutionary gradient here: the main fibrillar collagens of molluscs are similar to those of fish, the least evolved vertebrate class, whose connective tissue shares more chemical and physical characteristics with molluscan than mammalian connective tissue.

The integument of all species studied reacted with anti-rat collagen $\mathrm{V}$ antibody; the localization of this reactivity was closely similar to that of anti-type I-like collagen antibody from Sepia. This finding suggests the ubiquitous presence in molluscs of a minor collagen similar to type V, which may be associated with type I collagen, just as occurs in the heterotypic collagen fibrils of the human dermis. An alternative explanation is that the molluscan type I-like collagen possesses epitopes in common with vertebrate type $\mathrm{V}$ collagen. However the existence of two distinct types of molecules is supported by the findings of Rigo et $a l$. (paper submitted for pubblication) who isolated two distinct collagen molecules from the cartilage matrix of Sepia officinalis: a type I-like collagen and a collagen similar to vertebrate type V/XI. Type V-like collagens have also been found in several other invertebrate phyla (Tillet et al., 1996; Miura and Kimura, 1985; Yoshinaka et al., 1990) suggesting that the type $V$ collagen of invertebrates, which has remained largely unchanged in the course of evolution, is the ancestral collagen from which other types evolved, being required for basic functions such as collagen fibril assembly and growth.

Although type VI collagen is well documented in vertebrates (von der Mark et al., 1984; Keene et al., 1988; Keene et al., 1997) a similar collagen has not been reported before in invertebrates. Our experiments using antibody against human type VI collagen indicate the presence of a type VI-like collagen in the SEC of molluscan integument, with a diffuse distribution that recalls the similarly diffuse distribution of type VI collagen in the mammalian dermis. However, our electron microscope studies failed to reveal structures morphologically similar to mammalian microfilaments, which in mammalian dermis are largely composed of type VI collagen (Keene et al., 1988; Keene et al., 1997).

We observed no reaction to antibodies to human type III collagen in any of the molluscan tissues we studied, and hence conclude that this typical mammalian dermis collagen is absent from molluscs; it has not, to our knowledge, been reported in any other invertebrate.

The laminar shape and typical localization picked out by specific antibodies clearly correspond to the 
basement membranes identified by our parallel ultrastructural studies. In fact the basement membrane was the most morphologically diversified of all the SEC structures encountered in the molluscs we examined (Bairati et al., 2000; Bairati et al., 2001; Bairati et al., in preparation) and the immunostaining obtained with antibodies against several basement membrane components varied, from species to species, accordingly.

Thus, the positivity to anti-human type IV collagen antibody in areas corresponding to basement membrane found in all the molluscs examined constitutes further evidence that this collagen is widely distributed in all animal phyla, from the porifera upwards (Boute et al., 1996), and that its structural characteristics have remained largely unaltered through phylogenetic evolution (Pedersen, 1991; Cecchini et al., 1987; Fessler and Fessler, 1989; Exposito et al., 1994; Kramer, 1994).

We obtained reactions to anti-human collagen IV and anti-rat laminin in Sepia subepidermal basement membrane only after treating the sections with pepsin. This was almost certainly due to the very dense structure of the lamina densa (Bairati et al., in preparation) which would impede antibody access to the epitopes, and which was partly disrupted by the enzyme. Limited proteolysis is often successful in exposing epitopes for immunostaining (Finley and Petrusz, 1982; Wakamatsu et al., 1997).

Invertebrate species from various phyla have been shown to express immunoreactivity to vertebrate laminin antibody (Spiegel et al., 1983; Wessel et al., 1984; Vanhems and Delbos, 1989) and laminin-like molecules have been isolated from the ECM of several invertebrates (Mc Carthy et al., 1987; Fessler et al., 1987; Beck et al., 1989; Chiquet et al., 1988). A laminin-like molecule has also been reported in gastropods (Miller and Hadley, 1991; Humbert-David et al., 1995); our data show it is present in several molluscan classes.

We used a polyclonal antibody against laminin 1 from the basement membrane of the murine EHS tumor line which recognises epitopes present in all three laminin 1 chains $(\alpha 1, \beta 1, \gamma 1)$. Since other laminin isoforms (laminin 2, 3, 4, 6 and 7) contain one of the laminin 1 chains, we cannot determine which laminin isoforms are present in the basement membrane of the molluscs studied.

Our findings of positivity to antibodies against nidogen and the sugar portion of HSPG indicate the presence of similar components in the basement membranes of molluscs. The lack of reactivity to antibody against the protein portion of rat HSPG is presumably related to the fact that this is a highly variable and species-specific part of the molecule. HSPG has been localized by immunofluorescence in the basement membrane of the sea urchin (Wessel et al., 1984), but we are unaware of data on the immunolocalization of nidogen in invertebrates. Nevertheless the presence of nidogen in molluscan tissues is supported by the fact that laminin/nidogen-like complexes have been observed under the electron microscope in extracts from tissues of sea urchin Spherechinous granularis, leech Hirudo medicinalis (Beck et al., 1989) and marine sponge Oscarella tuberculata (Humbert-David and Garrone, 1993).

Our finding that molecules very like type IV collagen, laminin, nidogen and HSPG are all present in the basement membrane of molluscs confirms that the presence of all is required to form the three-dimensional framework constituting the basic architecture of the basement membrane, as proposed for mammalian basement membrane (Yurchenko and O'Rear, 1994; Timpl, 1996). This architecture would seem, therefore, to have appeared very early in evolution and to have remained remarkably constant.

In the SEC of the two terrestrial gastropods, immunostaining with antibodies against basement membrane components did not pick out a positive band below the epithelium, but extensive areas of positivity within the SEC. This is fully consistent with our electron microscopic findings (Bairati et al., 2001) which revealed at best a very thin lamina densa below the epithelium, not always discernible under the light microscope, and the presence within the SEC of extensive areas having similar ultrastructure to lamina densa, which surrounded SEC structures and extended to the deeper muscular stroma. That these extensive tracts turned out to be positive for type IV collagen, laminin, nidogen and HSPG provides convincing support to our suggestion, advanced on the basis of morphological data (Bairati et al., 2001) that these can be considered enormous tracts of basement membrane with, presumably, specific mechanical functions. Pedersen (1991) proposed that such extracellular subepithelial structures, together with typical basement membranes, should be considered as a single morpho- 
functional entity, the basement matrix, mediating relations between ecto-endoderm and mesenchyme derivatives.

Positivity to anti-HSPG antibody was found in the SEC of all the molluscs examined and this is consistent with our electron microscope observations (Bairati et al., 2000; Bairati et al., 2001; Bairati et $a l$. , in preparation) of abundant proteoglycan aggregates at the same site, and the proposal that various types of proteoglycans are ubiquitously present in all invertebrate phyla (Mathews, 1975b; Manfredi Romanini and Bolognani Fantin, 1992).

In this study we examined only the components considered necessary for forming the fundamental structures of the integumental extracellular matrix. Therefore we cannot exclude that other components typical of the extracellular matrix of vertebrate dermis (particularly other types of collagens), may be present as homologous or similar components also of the integumental extracellular matrix of molluscs.

To conclude, our comparative immunohistochemical study has demonstrated that integumental SEC is made up of components whose basic composition is similar in all the classes of molluscs investigated, although the ultrastructural and microscopic arrangement of these components varies, not only between classes but also between different species in the same class, in relation to varying anatomical and functional characteristics. Nevertheless the SEC of all species has components in common with other molluscan tissues, notably muscle stroma (Takema and Kimura, 1982) and cartilage (Bairati et al., 1999). Comparison of our findings with the situation in mammalian dermis is less straightforward as the latter is characterized by a much greater variety of molecular components. The composition and structure, however, of the basement membrane are similar in both mammals and molluscs, while the overall structure of the SEC differs. For the first time, evidences were gathered for the existence of collagen type VI and nidogen in the extracellular matrix of invertebrates.

\section{ACKNOWLEDGEMENTS}

Financial support was provided by funds from MURST ex $60 \%$. We thank Dr. Don Ward for the help with the English version. We are deeply grateful to Dr. R. De Sanctis and to the staff of the
Stazione Zoologica ‘A. Dornh' of Naples for supplying the marine animals.

\section{REFERENCES}

Bairati A.: The collagens of the Mollusca. In Biology of invertebrate and lower vertebrate collagens (Eds. Bairati, A. and Garrone, R.), Plenum Press, New York and London, pp. 277-297, 1985.

Bairati A., Comazzi M., Gioria M., Hartmann D. J., Leone F., and Rigo C.: Immunohistochemical study of collagens of the extracellular matrix in cartilage of Sepia officinalis. Eur. J. Histochem. 43, 221-225, 1999.

Bairati A., Comazzi M., and Gioria M.: An ultrastructural study of connective tissue in mollusc integument: I. Bivalvia. Tissue \& Cell 32, 425-436, 2000.

Bairati A., Comazzi M., and Gioria M.: An ultrastructural study of connective tissue in mollusc integument: II. Gastropoda. Tissue \& Cell 33, 426-438, 2001.

Beck K., McCarthy R.A., Chiquet M., Masuda-Nakagawa L., and Schlage W.K.: Structure of the basement membrane protein laminin: variations on a theme. In Cytoskeletal and extracellular proteins, (Eds. Aebi, U. and Engel, J.), Springer-Verlag, Berlin, pp. 102-105, 1989.

Boute N., Exposito J.Y., Boury-Esnault N., Vacelet J., Noro N., Miyazaki K., Yoshizato K., and Garrone R.: Type IV collagen in sponges, the missing link in basement membrane ubiquity. Biol. Cell. 88, 37-44, 1996.

Brocco S.L.: The ultrastructure of the epidermis, dermis, iridophores, leucophores, and chromatophores of Octopus dofleini martini (Cephalopoda: Octopoda). Dissertation, University of Washington, Seattle, 1976.

Cecchini J.P., Knibiehler B., Mirre C., and Le Parco Y.: Evidence for a type-IV-related collagen in Drosophila melanogaster. Evolutionary constancy of the carboxyl-terminal noncollagenous domain. Eur. J. Biochem. 165, 587-593, 1987.

Chiquet M., Masuda-Nakagawa L., and Beck K.: Attachment to an endogenous laminin-like protein initiates sprouting by Leech neurons. J. Cell Biol. 107, 1189-1198, 1988.

Démarchez M., Hartmann D.J., Herbage D., Ville G., and Pruniéras M.: Wound healing of human skin transplanted onto the nude mouse. II. An immunohistological and ultrastructural study of the epidermal basement membrane zone reconstruction and connective tissue reorganization. Devel. Biol. 121, 119-129, 1987.

Exposito J.Y., Suzuki H., Geourjon C., Garrone R., Solursh M., and Ramirez F.: Identification of a cell lineage-specific gene coding for a sea urchin $\alpha_{2}$ (IV)-like collagen chain. J. Biol. Chem. 269, 13167-13171, 1994.

Fessler J.H., and Fessler L.I.: Drosophila extracellular matrix. Ann. Rev. Cell Biol. 5, 309-339, 1989. 
Fessler L.I., Campbell A.G., Duncan K.G., and Fessler J.H.: Drosophila laminin characterization and localization. J. Cell Biol. 105, 2383-2391, 1987.

Finley J.C.W., and Petrusz P.: The use of proteolytic enzymes for improved localization of tissue antigens with immunocytochemistry. In Techniques in immunocytochemistry (Eds. Bullock, G.R. and Petrusz, P.), Academic Press, London, pp. 239-249, 1982

Humbert-David N., and Garrone R.: A six-armed, tenascinlike protein extracted from the Porifera Oscarella tuberculata (Homosclerophorida). Eur. J. Biochem. 216, 255-260, 1993.

Humbert-David N., Chandrasekaran S., Tanzer M.T., and Garrone R.: Laminin extraction and disorganization of collagen fibrils in snail muscles by EDTA treatment. Biol. Cell. 83, 3947, 1995.

Keene D.R., Engvall E., and Glanville R.W.: The ultrastructure of type VI collagen in human skin and cartilage suggests an anchoring function for this filamentous network. J. Cell Biol. 107, 1995-2006, 1988.

Keene D.R., Marinkovich M.P., and Sakai L.Y.: Immunodissection of the connective tissue matrix in human skin. Microsc. Res. Tech. 38, 394-406, 1997.

Kimura S., Takema Y., and Kubota M.: Octopus skin collagen: isolation and characterization of collagen comprising two distinct $\alpha$ chains. J. Biol. Chem. 256, 13230-13234, 1981.

Kramer J.M.: Structures and functions of collagens in Caenorhabditis elegans. FASEB J. 8, 329-336, 1994.

Manfredi Romanini M.G., and Bolognani Fantin A.M.: Polysaccharides and glycoconjugates in invertebrates. In Handbuch der histochemie (Eds. Graumann, W.), Gustav Fischer Verlag, Stuttgart-Jena-New York, pp. 1-211, 1992.

Mathews M.B.: Connective tissue: macromolecular structure and evolution. In Molecular biology biochemistry and biophysics (Eds. Kleinzeller, A., Springer, G.F. and Wittmann, H.G.), Springer-Verlag, Berlin-Heidelberg-New York, pp. 5863, 1975a.

Mathews M.B.: Connective tissue: macromolecular structure and evolution. In Molecular biology biochemistry and biophysics (Eds. Kleinzeller, A., Springer, G.F. and Wittmann, H.G.), Springer-Verlag, Berlin-Heidelberg-New York, pp. 115, 1975b.

Mc Carthy R.A., Beck K., and Burger M.M.: Laminin is structurally conserved in the sea urchin basal lamina. EMBO J. 6, 1587-1593, 1987.

Miller J.D., and Hadley R.D.: Laminin-like immunoreactivity in the snail Helisoma: involvement of a $300 \mathrm{kD}$ extracellular matrix protein in promoting outgrowth from identified neurons. J. Neurobiol. 22, 431-442, 1991.

Miura S., and Kimura S.: Jellyfish mesoglea collagen. Characterization of molecules as $\alpha_{1} \alpha_{2} \alpha_{3}$ heterotrimers. J. Biol. Chem. 260, 15352-15356, 1985.

Pedersen K.J.: Structure and composition of basement membranes and other basal matrix systems in selected inverte- brates. Acta Zool. 72, 181-201, 1991.

Rigo C., Hartmann D.J., and Bairati A.: Electrophoretic and immunochemical study of collagens from Sepia officinalis cartilage. (In press)

Sage H., and Gray W.R.: Studies on the evolution of elastinI. Phylogenetic distribution. Comp. Bioch. Physiol. 64, 313328, 1979.

Spiegel E., Burger M.M., and Spiegel M.: Fibronectin and laminin in the extracellular matrix and basement membrane of sea urchin embryos. Exp. Cell Res. 144, 47-55, 1983.

Takema Y., and Kimura S.: Two genetically distinct molecular species of octopus muscle collagen. Biochim. Biophys. Acta 706, 123-128, 1982

Tillet E., Franc J.M., Franc S., and Garrone R.: The evolution of fibrillar collagens: a sea-pen collagen shares common feature with vertebrate type V collagen. Comp. Biochem. Physiol. 113, 239-246, 1996.

Timpl R.: Macromolecular organization of basement membranes. Curr. Opin. Cell Biol. 8, 618-624, 1996.

Vanhems E., and Delbos M.: Ultrastructural localization of lectin-binding sites and laminin-like immunoreactivity in glial cells and neurites growing out from explant cultures of the central nervous system of embryonic locusts. Cell Tissue Res. 258, 429-436, 1989.

Vialle-Presles M.J., Hartmann D.J., Franc S., and Herbage D.: Immunohistochemical study of the biological fate of a subcutaneous bovine collagen implant in rat. Histochemistry 91, 177-184, 1989.

von der Mark H., Aumailley M., Wick G., Fleischmajer R., and Timpl R.: Immunochemistry, genuine size and tissue localization of collagen VI. Eur. J. Biochem. 142, 493-502, 1984.

Wakamatsu K., Ghazizadeh M., Ishizaki M., Fukuda Y., and Yamanaka N.: Optimizing collagen antigen unmasking in paraffin-embedded tissues. Histochem. J. 29, 65-72, 1997.

Wessel G.M., Marchase R.B., and McClay D.R.: Ontogeny of the basal lamina in sea urchin embryo. Dev. Biol. 103, 235245, 1984.

Yoshinaka R., Mizuta S., Itoh Y., and Sato M.: Two genetically distinct types of collagen in kuruma prawn Penaeus japonicus. Comp. Biochem. Physiol. B 96, 451-456, 1990.

Yurchenco P.D., and O'Rear J.J.: Basal lamina assembly. Curr. Opin. Cell Biol. 6, 674-681, 1994.

Zylberberg L., Bonaventure J., Cohen-Solal L., Hartmann D.J., and Bereiter-Hahn J.: Organization and characterization of fibrillar collagens in fish scales in situ and in vitro. J. Cell Sci. 103, 273-285, 1992. 